\title{
CONRAD DETREZ - LA BELGITUDE EN «JE» ET EN JEU
}

\begin{abstract}
Pantkowska Agnieszka, Conrad Detrez - la belgitude en «je» et en jeu [Conrad Debrez the Belgilude as „I" and as a game]. Studia Romanica Posnaniensia, Adam Mickiewicz University Press, Poznań, vol. XXX1: 2004, pp. 69-82. ISBN 83-232-1353-4, ISSN 0137-2475.
\end{abstract}

Conrad Debrez is one of those Belgian writers in whom "the Belgitude" (being Belgian), or to put it differently, a relation to Belgium, characteristic of the writers of the 70 s of the 20 th century, is full of contradictory and intermingled feelings and emotions, from love to disdain, has found its fullest expression.

It is suggested that in his case, the attitude to homeland, and therefore, to his own identity, should be traced on the basis of his autobiographical trilogy where a peculiar play with ,I" is in fact an attempt at solving the problem of a Belgian's identity.

Conrad Detrez jouit d'un statut privilégié dans le contexte de la décennie 1974 -1985 , qualifiée par la critique littéraire belge de période de la belgitude ${ }^{1}$. En effet, le cadre temporel relatif au phénomène de la belgitude, recouvre celuj de la vie «littéraire» de Conrad Detrez. De fait, né en 1937, cet écrivain publie son premier texte romanesque ${ }^{2}$, Ludo, en 1974. Suivent Les Plumes du coq (1975), L'Herbe à brûler (1978), La Lutte finale (1980), Les Noms de la tribu (1981) et La Mélancolie $d u$ Voyeur publié un an après la mort de l'auteur survenue en 1985.

La coïncidence temporelle n'est pas anodine.

Cette nouvelle sensibilité, que Pierre Mertens définit comme belgitude, est admirablement circonscrite dans et par un ouvrage collectif sans précédent intitulé: La Belgique malgré tout dans lequel Detrez apporte sa contribution. Il écrit à cet effet une pièce radiophonique de politique-fiction (l'action se passe le 31 décembre 1999 au moment où le roi décide de proclamer la république en présence des représentants du pays). Elle s'intitule Le dernier des Wallons et dit, sur le mode ,

' $L e$ terme de la belgitude est forgé sur le modèle de la négritude; il définit, en creux, le rapport du pays francophone à la langue et à la culture française. Ce terme est promu par Picrre Mertens et Claude Javeau dans un numéro spécial des «Nouvelles litteraires» intitulé L'Autre Belgique en 1976.

${ }^{2}$ II a publié avant Pour la libération du Brésil, texte interdit par le ministère français de l'intérieur (1970), Les Mouvements révolutionnaires en Amérique latine (1972), et de nombreux articles de presse. 
burlesque, l'éclatement de la Belgique et le mal identitaire auquel les francophones doivent faire face. Remarquons par ailleurs que Detrez prend volontiers la plume pour se prononcer au sujet de la Belgique, de sa situation socio-politique, de l'actualité qui la trouble. Évoquons, à titre d'exemple: Les Wallons, les Flamands et l'Europe, article paru dans «Esprit» en 1968, Une Belgique fabuleuse, publié dans «Télérama» en 1979, ou Une fureur de Wallon paru dans «Le Monde» en 1979. Dans ces divers articles où l'ironie s'avère son arme redoutable, digne d'un exguérillero du Brésil, Detrez critique la politique du pays unitaire, dénonce les paradoxes belges et s'exprime en tant que Wallon ${ }^{3}$. La reconnaissance explicite de son origine va cependant de pair avec un malaise dont témoigne la pièce Le dernier des Wallons. L'accent mis sur le mot «dernier» introduit une impression de finitude, une touche apocalyptique dont son cuvre entière est d'ailleurs stigmatisée. Ce malaise se manifestera de façon péremptoire, en 1982, lorsque Conrad Detrez à qui la critique a collé l'étiquette d'écrivain wallon, avant même de le qualifier de belge, choisira et adoptera la nationalité française. Ce qui permet à certains de situer Detrez au même niveau que Michaux ou Simenon, leur a-belgité étant le dénominateur commun: «L'on chercherait en vain une culture belge dans l'œuvre de nos grands artistes, nos peintres de Magritte à Alechinsky, nos écrivains, presque tous émigrés d'ailleurs en France: Henri (sic! $)^{4}$ Bauchau, Dominique Rollin (sic!) ${ }^{5}$ (...) René Kalisky ou Conrad Detrez qui mourut français» ${ }^{6}$.

Évidemment, c'est une constatation fort tendancieuse, bien que le cosmopolitisme de l'œuvre detrezienne ne puisse pas être contesté. Elle est en même temps révélatrice des divergences qui surviennent dans la définition de cet écrivain. La difficulté à le classer reflète la difficulté à se définir éprouvée par Detrez dont l'ceuvre entière sera orientée vers la recherche de son identité problématique.

La contradiction entre cette constatation et l'engagement «belge» de Detrez visible dans ses articles de presse, n'est qu'apparente. En fait, il s'agit ici de la suite logique de cause à effet, où la reconnaissance de son identité belge, wallonne n'est conquise qu'à la suite d'un long processus de recherche opérée dans et par l'écriture de ses premiers textes littéraires. Conquise, certes, mais est-elle également acceptée? Il parait évident que l'année 1982 constitue un aboutissement, exprimé par la négation, de la quête identitaire. Detrez s'en est expliqué dans son dernier livre, inachevé, La Mélancolie du voyeur.

En commentant le second livre de Detrez, Les Plumes du coq, Pierre Mertens a déclaré en 1975: «Je n'en connais guère qui assument avec autant de santé, une telle

${ }^{3}$ «Wallon, je souhaite un fédéralisme à l'abri des outrances qui menacent aujourd'hui», C. Detrez, Les Wallons, les Flamands et l'Europe, cité d'après le dossier établi par A.J. Dubois dans la Lecture de Ludo, Labor, Bruxelles 1988, p. 191.

${ }^{4}$ Bauchau signe ses texte Henry.

${ }^{5}$ La graphie correcte du nom de Dominique est Rolin.

${ }^{6}$ Culture française ou «culture belge»? page Web de la Maison de la francité. 
absence de complexe, leur «belgitude»» ${ }^{7}$. Detrez n'est pas là, semble-t-il, pour le contredire lorsqu'il écrit, en 1979:

La Belgique mammifère amuse, dit-on, les Français. (...) La France pourtant a ses côtés veau. Mais le veau français est trop sage. Il est éduqué, il raisonne, il possède la glande pinéale. Et il ne patauge pas autant que son voisin dans l'cau. Le veau belge croil utile de calquer le français. Il a tort. Jamais il n'arrivera à larmoyer aussi artistement que Julien Green ou François Mauriac. Jamais il ne sera, comme à Paris, une bête élégantc.

Le veau belge doit rester un malappris, un naif. Il doit courir après sa queue jusqu'à la fín des temps. De cette insistance naît l'attitude métaphysiquc. Le Belge y excelle. C'est sa gloire. La Belgique, comme dans les légendes, est un animal fabuleux ${ }^{8}$.

Cet extrait de l'article intitulé Une Belgique fabuleuse abonde en expressions et toumures ironiques à l'adresse du pays. Le fait que Detrez qualifie p.ex. le Borinage de Paysage du Néant, ou qu'il affirme que «la patrie de Rubens n'existe plus» ${ }^{9}$ est révélateur du rapport de l'écrivain à la Belgique, pays dont l'existence réelle est mise en doute ce qui situe la Belgique quelque part entre absence et magie.... Comme le souligne dans la lecture de Ludo André-Joseph Dubois, la Belgique reste pour l'auteur le pays indicible, certes, mais qui engendre en même temps le besoin de se dire. J'y reviendrai.

Indicible? et qui doit se dire? Rien alors d'étonnant que l'auteur cherche à trancher ce noud belge; pour ne pas dire: gordien, avec l'épée de son écriture. Naissent: Ludo, les Plumes du coq et L'Herbe à brûler, trilogie initiatique, sorte de Bildungsroman ${ }^{10}$ dont chaque volet renvoie à une étape de la vie, notamment à l'enfance, l'adolescence et à la jeunesse. L'ordre chronologique de la vie humaine que la trilogie observe, sous-entend une recherche méthodique, une progression dans l'élucidation de l'objectif imposé par la nature du récit personnel: se comprendre, reprendre le contrôle de sa vie.

Le fait que cet écrivain entre en littérature en publiant un récit d'enfance est significatif. Tout en renouant avec ce que Jacques Lecarme, grand spécialiste français de l'autobiographie, considère comme une spécialité belge, un tel choix trahit le besoin de l'auteur de remonter aux origines afin de mieux comprendre l'être qu'il est devenu. Simultanément il semble suggérer que la source de son mal quel que soit le nom qu'on lui donne, est originelle, indépendante de la volonté, bonne ou mauvaise, de l'homme. Detrez victime alors? telle semble la conviction de William Cliff, poète et ami de Detrez, lorsqu'il écrit:

${ }^{7}$ P. Mertens, L'attente de l'aube, «Le Soir», le 1.10.1975.

${ }^{8}$ C. Detrez, Une Belgique fabuleuse, dans «Télérama», le 9 mai 1979, cité d'après le dossier établi par A.J. Dubois dans la Lecture de Ludo, op. cit., p. 192-195.

${ }^{9}$ Idem.

${ }^{10}$ Terme employé et commenté par Jean-Marie Klinkenberg dans sa lecturc de L'Herbe à brûler, Labor, Bruxelles 2003, p. 321. 
Sainte Marie de Jésus toi qu'on a

Inlassablement invoquée au long

Des longs siècles de chrétienté tu n'as

Pas été sourde à ce petit wallon

Quand sa prière humble vers toi s'allon-

Geait à travers une enfance martyre

Dame du Ciel reine des souvenirs

Accueille-le dans ton coeur innombrable

Et qu'il y trouve une eau meilleure à boire

Que celle bue au moment de mourir".

Il paraît probable que Detrez aurait partagé cette opinion; quatre ans après la publication de Ludo il écrit:

J'ai cherché à connaître les causes de ces faillites, à mettre à nu les racines de mes révoltes.

J'ai lu Freud et j'ai commencé la plume à la main, à me livrer à une sorte d'analyse. Ainsi est né mon premier roman ${ }^{12}$.

Conrad Detrez a qualifié ces trois romans d' «autobiographie hallucinée»" 1 . D'emblée le problème du genre se pose, car ces deux termes sont parfaitement antinomiques. En même temps cependant se manifeste cette oscillation si caractéristique pour cet écrivain du perpétuel entre-les-deux qui veut dire l'indicible... et qui y arrive, par la voie de la négation.

Si l'on reprend la définition, canonique, de Philippe Lejeune, l'autobiographie est un récit rétrospectif en prose qu'une personne réelle fait de sa propre existence, quand elle met l'accent sur sa vie individuelle, sur l'histoire de sa personnalité. Hallucination par contre relève du domaine des fantaisies, de l'imaginaire, bref: connote la fiction romanesque.

On peut pousser l'interprétation plus loin cependant: hallucination suggère un hors du naturel: que sa cause soit une maladie ou qu'elle soit provoquée par un excitant, l'hallucination est la manifestation d'un subconscient que la raison ne contrôle pas, alors que l'autobiographie ambitionne de reprendre le contrôle justement de la vie, d'y imposer l'ordre. Acusi, ce que le terme d'autobiographie cherche à clarifier, élucider, le terme d'hallucination le brouille. Est-ce donc une impasse? Serge Doubrovsky, en publiant Le Fils en 1977 a donné un nom à cette forme hybride: l'autofiction. Selon Doubrovsky, l'autofiction serait donc une auto-

${ }^{11}$ W. Cliff, Conrad Detrez, Dilettante, 1990.

${ }^{12}$ C. Detrez, Le jardin de ma vie, «Le Figaro», le 21 nov. 1978, cité d'après le dossier établi par A.J. Dubois dans la Lecture de Ludo, op, cit., p. 198.

${ }^{13}$ Cette information se trouve au dos de la couverture de L'Herbe à brûler: «Après Ludo et Les Plumes du coq, Conrad Detrez nous donne ici un nouvel episode de son «autobiographie hallucinée». Sachant que le quatrième de couverture est confié d'habitude à l'auteur, cet aveu fonctionne comme une sorte de pacte avec le lecteur tout en programmant sa lecture des textes précédents, qualifiés pourtant de «romans». Notons par ailleurs qu'A.-J. Dubois prouve, dans la Lecture de Iudo, que la trilogie n'est pas un fruit de hasard, mais le résultat du projet conscient de l'auteur (op. cit., p. 166). 
biographie (au sens lejeunien du terme) fictive que quelqu'un donne de son existence en visant des objectifs psychanalytiques. Certains chercheurs veulent y voir, à tort, tout simplement un roman autobiographique. Toutefois nombreuses sont les différences, à commencer par l'attitude de l'auteur face à son texte. Dans un roman autobiographique l'auteur se cache derrière un «il» qui, bien qu'il témoigne d'une ressemblance parfois même étroite avec l'auteur, s'en distancie pourtant, et l'auteur ne fait que prêter à son personnage, qui est autre que lui-même, certains traits et/ou faits de sa vie. L'auteur de l'autofiction endosse ouvertement la responsabilité de son texte en respectant la condition sine qua non de l'autobiographie: l'identité de l'auteur - narrateur - personnage prịncipal. Il ne se distancie pas de son moi, mais de la vision, de la relation de sa vie. Il le signale par l'inscription de «roman» en sous-titre sur la couverture fondant ainsi le pacte romanesque, attestation de la fictivité. Il donne une autobiographie hallucinée, en somme...

Qu'il s'agisse, chez Detrez, de l'autofiction, tout au moins dans le cas de ses premiers livres, ne fait pas aujourd'hui l'ombre d'un doute. (Detrez, innovateur malgré lui, inspire un autre grand écrivain belge dont les écrits amènent Philippe Lejeune à revoir sa théorie. Il s'agit de William Cliff, déjà mentionné, auteur de son autobiographie en vers, auteur également de la biographie en vers de Conrad Detrez ${ }^{14}$.)

L'auteur l'indique explicitement lorsqu'il écrit en 1980: «J'éprouve trois besoins: le besoin de me connaitre, d'exorciser mes démons, de découvrir les racines de mes rebellions, d'où auto-analyse, d'où vertu thérapeutique de l'écriture, d'où catharsisme» ${ }^{15}$. Son œuvre serait ainsi un modèle du genre d'autant plus intéressant que chez lui le terme d'hallucination semble plus proche de transformation onirique. Chez Detrez ce n'est donc pas la fiction gratuite, effet de quelque caprice ou recherche d'effets littéraires, qui prend le dessus, mais le factuel voilé, fantasmé, masqué (par peur? pudeur?) ou peut-être résultant de l'impossibilité de se dire, néanmoins issu de la pulsion de l'auteur à se découvrir. Un critique a observé non sans raison que «c'est une autobiographie qui ne veut pas dire son nom» ${ }^{16}$. Pourquoi?

D'une part il me semble qu'il faut chercher la raison de cette attitude dans le sentiment profond de la culpabilité que l'auteur éprouve, culpabilité au sens religieux même du terme. Petit séminariste à Saint-Remy, habitué du confessionnal et de l'examen de conscience, Detrez semble conférer à toute son cuvre un caractère de confession. Cette impression est accrue par la dimension apocalyptique que la présence récurrente de la mort confère à chaque récit personnel de Detrez. En effet, Conrad Detrez pouvait se sentir coupable d'avoir trahi sa famille, son

\footnotetext{
${ }^{14}$ Biographie hétérogène d'ailleurs car pour parler de la vie de son ami, Cliff part de ses propres récits autobiographiques.

${ }^{15}$ «Le Monde», le 23.03.80, cité à après le dossier établi par A.J. Dubois..., op. cit.

${ }^{16}$ Le Cerisier du 15 février 1979, cité à après le dossier établi par A.J. Dubois..., op. cit.
} 
éducation, sa religion, son pays enfin. Ses préférences homosexuelles n'y seraient pas sans importance non plus.

Nombreux sont les indices qui attestent de la faute obscure qui inquiète le protagoniste. Dans Ludo le petit narrateur se sent coupable envers sa mère qu'il fuit car elle l'ennuie, qu'il n'obéit pas, qu'il tue symboliquement à la fin du livre, car elle s'impose comme obstacle au développement de sa personnalité. Le sentiment de pêché, conjugué à la honte, accompagne également et surtout la découverte de sa sexualité: «Elle (ma mère) me gifle, crache sur mon sexe, le serre, elle va me le couper, c'est fini, c'est la dernière fois, je ne sais 'pas cacher ma honte et ma misère..." ${ }^{17}$.

Dans Les Plumes du coq la notion de pêché est inséparable de la sexualité, acquerrant cette fois-ci une dimension mystique; la vision de l'Époux dénudé sème des troubles sensuels chez l'adolescent; son apparition met fin au premier baiser donné à une jeune fille et ressenti comme une faute grave. Dans L'Herbe à brûler le pêché devient tout à fait corporel: le baiser d'Alphonsine que le jeune homme considère comme un pêché: «ll était grand mon pêché; c'était comme une bête à plusieurs têtes et tentacules de toutes les grosseurs" ${ }^{18}$. Apparaît également la culpabilité liée aux choix politiques (déplumage du coq, symbole wallon, soit le reniement de son camp pendant la question royale), qui aura son prolongement dans L'Herbe à brûler. L'idée d'avoir mal choisi ou trahi la cause, ne serait-ce qu'un moment, devient l'un des thèmes des Les Plumes du coq et de L'Herbe à brûler. Comparons:

Coincé entre les deux groupes, ballotté, bousculé par les uns puis par les autres, je luttais pour me dégager, pour imposer mes arguments, je luttais pour ne pas perdre de vue l'ennemie aux regards de velours (...) Lorsque les femmes sont passées des paroles aux actes, j'ai préféré garder mes convictions pour moi, j'ai jouć les muets. (...) Je n'ai eu d'autres recours que de m'emparer d'une pancarte gisant sur le sol avec sa victime et, toujours sans prolérer la moindre opinion et souriant aux femmes, de me frayer un passage jusqu'à la jeunc fille. (...) - Mademoiselle! Mademoiselle! (...) - C'est uoui» ou c'est «non»? - C'est ... «non», m'écriais-je, c'est «non» ${ }^{19}$.

avec un passage de L'Herbe à brûler:

J'ai manqué défaillir; je m'étais sans le savoir fourvoyé parmi mes ennemis, je me trouvais incorporé à une bande de contre-manifestants. Pour ce faire (rejoindre son groupe) il convenait de me glisser dans le premier rang de mes adversaires. (...) J'étais parvenu au premier rang quand le choc s'est produit. Attaquants et contre-attaquants sont tombés les uns sur les autres. Un gourdin m'a frôlé la tempe ct arraché l'oreille. J'ai tourné sur moi en criant; «Je suis des vôtres, je suis des vôtres!» mais en vain: un autre gourdin s'est abattu sur moi ${ }^{20}$.

\footnotetext{
${ }^{17}$ C. Detrez, Ludo, Labor, Bruxelles 1988, p. 45.

${ }^{18}$ Idem, $L^{\prime}$ Herbe à brûler, Calmann-Lévy, Paris 1978, p. 48.

${ }^{19}$ Idem, Les Plumes du cog, Labor, Bruxelles 1975, p. 106.

${ }^{20}$ Idem, L'Herbe..., op. cit., p. 76.
} 
Confondre les camps revient à se confondre, à perdre ses repères. Ainsi, le cri désespéré du narrateur «je suis des vôtres» est en fait une manifestation du besoin de se définir, de trouver sa place dans la réalité qui l'entoure. Toutefois, dans une Belgique divisée par des antagonismes religieux, politiques, linguistiques et sociaux, trouver des repères solides pour un homme qui se sent autre ${ }^{21}$, qui est en proie à une crise d'identité s'avère impossible. C'est sans doute une des raisons qui le pousseront à partir s'engager au Brésil, pays où la ligne de démarcation entre les deux camps est nette, où il est plus aisé de distinguer l'ami de l'ennemi.

L'expérience politique, nous l'avons vu, ne procure au protagoniste que l'amertume, la souffrance et la désillusion. Son engagement s'avère vain, voire absurde, car dépourvu de sens. Il le découvre grâce à deux collègues étrangers: Léopoldus, le jeune Congolais et surtout Rodrigo, le jeune Brésilien. C'est son regard neutre, car extérieur, qui permettra au narrateur de mesurer le caractère superficiel des préoccupations européennes:

Ton Europe, elle n'a rien à voir avec mon rêve, elle est presque bête, et gâtée, trop gâtée, elle n'invente plus rien, elle passe son temps à se diviser sur des trucs accessoires. (...) L'Europe de Jeanne d'Arc, bien sûr, ou celle de Pie VII, l'Europe d'hier. Mais celle d'aujourd'hui, qu'est-ce que c'est? Celle de ton pays, de tes évêques? Où vis-tu donc et à quclle époque, hein? (...) - dans des querelles de briques! Mille briques pour l'école du chanoine Machin (...) un autel en marbre toumé comme ceci pour le révérend préfet, un autel en albâtre tourné comme cela pour le directeur... Et tout cela au pays d'Erasme, au pays de Louvain-la-Grande: celle des bouquins, naturellement, car l'autre, celle d'aujourd'hui, Louvain-la-merde, on n'en parle pas et pourtant c'est dans Louvain-la-merde qu'on patauge! (...) Rodrigo exagère, me disais-je (...) Cependant je ne trouvais rien à répliquer et le silence, mon silence, il pouvait l'interpréter comme un acquiescement, une défaite ${ }^{22}$.

Le sentiment de culpabilité est indissociable de celui de l'échec que l'écriture tend à élucider. Dans le cas de Detrez, l'échec se situe à trois niveaux: politique, ainsi que nous venons de le voir, mais aussi amoureux et religieux; tous les trois étant étroitement liés au contexte belge.

L'expérience religieuse se solde, elle aussi, par un fiasco. Le personnage principal découvre que l'école catholique est le siège non seulement de la bêtise, de l'obscurantisme flagrant, mais également des vices et aberrations sexuelles. Comme le jeune homme engagé se fait battre, à cause d'un malheureux hasard, par ses condisciples, l'adolescent, catholique fervent, voire mystique, se fait violer par son supérieur. Jeune homme, il découvre par ailleurs le côté hermétique de l'enseignement religieux, incompatible avec la vraie vie, ainsi que l'hypocrisie du clergé. Révolté contre la rigidité du droit canonique qui n'admet pas l'adoration de Dieu en négligeant les rituels, le narrateur quitte le séminaire pour quitter, plus tard, la religion.

${ }^{21}$ Ses origines sociales peuvent également y joucr un rôle; il est né dans un milieu paysan, de mère flamande et de père wallon.

${ }^{22}$ C. Detrez, L'Herbe..., op. cit., p. 64-65. 
Il en est de même avec l'expérience amoureuse. Que ce soit avec une fille des Les Plumes du coq ou avec Alphonsine de L'Herbe à brâler, l'expérience amoureuse n'occasionne que les désillusions douloureuses. La jeune fille que l'adolescent croit «une âme d'élite, un ange, c'était Jeanne d'Arc» (p. 106) s'avère une fille simple, trop simple sans doute, tandis que le contact avec Alphonsine lui procure le dégoût tant physique que moral. Il est intéressant de remarquer à quel point le personnage féminin est doté de valeurs négatives. Si la jeune fille est un personnage impur, propre à inspirer le dégoût (surtout lors du cọtact charnel, son apparence étant plutôt agréable) la mère ne vaut pas mieux aux yeux du narrateur de $L u d o$ avant tout, mais également de celui des Les Plumes du coq et de L'Herbe à brûler où c'est surtout l'absence de la mère qui est contée sur le mode de culpabilité (elle est morte seule, solitaire, sans aucune nouvelle de son fils). La mère dans Ludo présente toutes les caractéristiques opposées à celles que la tradition attribue à la mère. C'est une femme bornée, ennuyeuse, agressive et brutale (elle bat son fils, lui donne des coups de pieds, lui arrache les cheveux). Elle séquestre le garçon physiquement en l'empêchant de sortir dans le jardin, (elle l'attache au lit par exemple) mais aussi psychiquement en lui arrachant le serment de ne jamais la quitter. Possessive, elle fait irruption dans le monde de la sensualité en train d'éclore du garçon:

Ma mère me lèche les joues, me caresse avec un coin de son tablier mais je pleure encore plus. Ça passera, elle dit que ça passera, c'est comme ça qu'on apprend, elle restera toujours avec moi, je grandirai, je prendrai la place de mon père. (...) Pourtant... non, ça ne se dit pas, je ne dois pas le lui dire que ce n'est pas avec elle que je veux me marier ${ }^{23}$.

Bref, pour reprendre le langage freudien: c'est une mère castratrice ${ }^{24}$. Première femme que le petit personnage connaisse, elle est à la source du désenchantement sur le féminin que le garçon étendra sur les femmes à venir, ce qui peut être considéré comme une explication de son homosexualité.

Le thème de la faute et de la culpabilité s'accompagne chez Detrez du thème du voyage qui revêt alors une forme de fuite.

En effet, l'auteur a passé une partie de sa vie au Brésil en prenant une part active à la dure réalité marquée par le régime totalitaire et le coup d'état militaire en 1964. Il a séjourné en outre au Portugal, en Algérie où il s'est engagé dans la lutte contre l'analphabétisme, en France finalement. Nulle part pourtant il ne trouve sa place. Vagabond ou banni, il confie: «Et moi, toujours à errer entre les décombres de mes vieilles croyances, les décombres du sexe, de la politique. Fuir, je devais fuir» ${ }^{25}$.

${ }^{23} \mathrm{Idem}$, Ludo, op. cit., p. 106.

24 "- Tu resteras toujours près de moi, n'est-ce pas? - On ne partira pas? - Si on doit partir, on nagera ensemble (...) - On ira dans le jardin? - (...) Non et non ! Pour encore te faire voler? Moi, je ne vole pas. Est-ce que je vole? - Je dis «non» mais j'ai envic de dire autre chose, je ne sais pas quoi», idem, Ludo, op. cit., p. 108.

${ }^{25}$ Idem, La Mélancolie du voyeur, Denoël, Paris 1986, p. 35. 
C'est dans son enfance que l'auteur cherche les origines de ce besoin d'évasion. La première fuite, combien symbolique, se manifeste par le meurtre de sa mère, car parmi tous ses vices l'un était insoutenable: elle s'impose comme un obstacle dans la quête de la liberté, symbolisée par le personnage de Ludo, ami du narrateur «qui lui ressemblait comme un frère» pour reprendre la célèbre formule de Musset. La mère destructrice règne dans la maison qui est dépourvue, elle aussi, de valeurs positives. Sombre et puante à cause de la fiente qui, mise en sacs, bouche les fenêtres, isolée, telle une île, par les eaux, barrière infranchissable pour le narrateur qui ne sait pas nager («elle n'a jamais voulu me l'apprendre. 'Tu nageras quand je ne serai plus, disait-elle, quand je serai passée de l'autre côté'» ${ }^{26}$ ) la maison devient le synonyme de prison: «Elle ne supporte plus de me voir jouer dans le jardin. Si je mets les pieds dehors, elle appelle au secours. (...) ma mère me ramène dans le corridor, m'enferme avec elle.... ${ }^{27}$. A l'opposé de la mère et de la maison-prison se trouve Ludo qui, lui, se meut dans un espace ouvert, dominé par le soleil. Comme la mère se définit par la maison, Ludo est maître du jardin dont la signification acquiert une dimension de jardin paradisiaque, édénique où il n'y a pas de fruit défendu.

Le jardin de Ludo est un alignement de guirlandes poudrées de bronze. (...) Le jardin de Ludo. Un jardin fou. Un jardin m'as-tu vu... Je crève sur mon île, je n'y tiens plus. Ma mère passe des journées entières sur son mur de sacs, elle complote. (...) Qu'elle complote, la mère ! J'ai mon plan, je passerai de l'autre côté. Je jetterai une passerelle entre ma prison et le jardin. Je courrai sur un pont fait de planches et de coussins, attaché avec des ficelles de caoutchouc. Je découperai les chambres à air ${ }^{28}$.

Si dans le premier livre la fuite n'est vécue que sur un mode intérieur, psychologique, dans Les Plumes $d u$ coq elle prend une forme plus concrète. Le protagoniste quitte sa famille, son village pour continuer l'éducation dans un collège catholique. L'espace s'élargit donc, le cercle fermé du foyer familial est rompu. Lui succède cependant le sentiment d'enfermement dû à la religion. Le collège, "cette bâtisse (...) massive, (...) uniformément brune, (entourée de) ces hauts murs, pierres et briques confondues» ${ }^{29}$, surveillé par des enseignants, organisé selon une discipline quasi militaire, c'est une autre prison, plus grande que la maison maternelle, mais pas plus ouverte qu'elle. Cet espace clos est aussi une source de souffrances, tant physiques que psychiques et morales et n'éveille qu'un désir: $s^{\prime}$ 'évader ${ }^{30}$. Le sentiment du pêché, les remords qui suivent font que la tentative

\footnotetext{
${ }^{26}$ Idem, Ludo, op. cit., p. 54.

${ }^{27}$ Idem, p. 48.

${ }^{28}$ Idem, p. 100 .

${ }^{29}$ Idem, Plumes..., op. cit., p. 21.

${ }^{30}$ "Vu de l'extérieur le pensionnat semblait voué à la décomposition. Pourquoi, me suis-je tout à coup demandé, n'avais-je jamais songé à m'en évader, à passer carrément de l'autre coté du mur, à me mettre en marche, en ligne droite, sans me retourner? pourquoi, au sortir du champ de betteraves,
} 
d'évasion se solde par un échec. La fuite prendra sa forme définitive dans L'Herbe à brûler. Éloigné de son village natal, du collège, le séminaire à Louvain constitue une étape dans l'affranchissement du protagoniste. Il se libérera de tout ce qui limite son esprit: religion, tradition, pour se libérer du poids le plus lourd, semble-til, de son pays qu'il quitte "comme on quitte, recru d'air vicié, un w.-c. public»" ${ }^{3 !}$.

Car, bien que le thème de la faute soit récurrent dans l'cuvre de Detrez, il serait imprudent d'y voir l'explication unique de ce refus de signer le pacte autobiographique engageant l'auteur à prendre la responsabilité du récit de sa vie.

Le genre que l'auteur choisit pour s'exprimer, l'insistance avec laquelle le «moi» imprègne l'œuvre ainsi que les mots durs qu'il adresse à son pays suggèrent qu'il faut chercher l'explication plus loin, dans la crise identitaire due à l'origine belge, dont la culpabilité et l'évasion ne sont que des dérivatifs.

Les trois romans organisés chronologiquement, constituent trois échelons qui mènent de la question «qui suis-je» à la réponse «je suis Belge». La quête identitaire s'avère donc inséparable de la quête du pays.

Dans Ludo l'identité du narrateur et du personnage principal n'est pas évidente; c'est Ludo, ami du narrateur qui focalise l'action; c'est lui qui donne d'ailleurs son nom au titre du livre. L'bétérogénéité du narrateur et du personnage n'est qu'apparente; nombreuses sont les indications qui autorisent à considérer ces deux personnages comme deux facettes d'un seul homme:

Les fugueurs s'étaient évanouis l'un contre l'autre. (...) Nous étions, parait-il, etendus face à face. (...) Les mères nous donnent des coups de pied. Les coups de pied nous rejettent l'un sur l'autre. Ma mère a serré le poing. Ludo l'évite de justesse. 11 me tombe dessus, nous entrons l'un dans l'autre, disparaissons dans un trou... ${ }^{32}$.

Toujours dans cette perspective, il faut évoquer également l'épisode avec une photographie représentant Ludo que la mère du narrateur tient pourtant cachée sous son oreiller et qui a été prise, de toute évidence, avec le narrateur pour modèle (voir pp. 76-78).

Il est intéressant de remarquer que l'identité du narrateur n'est jamais donnée, pas une seule fois, alors que celle de Ludo est déclinée à tout moment. Sa mère désigne le narrateur par un «tu» neutre ou par «gamin», «sale gamin» ou encore "gamin de merde». Il est à doserver que cette désignation qui fonctionne comme une marque de différenciation du narrateur s'applique également à Ludo.

Le narrateur se présente lors d'un jeu avec son ami comme Monsieur Moi, par la majuscule:

n'avais-je pas, plutôt que de revenir à ma prison, mis le cap sur la route, une route sans détours, sans montées ni descentes, tracée à l'écart des bourgs et des villages ct surtout du mien...?» Idem, Plumes, op. cit., p. 154.

${ }^{31}$ Idem, L'Herbe..., op. cit., p. 95.

${ }^{32}$ Idem, Ludo, op. cit., p. 40-41. 
Monsieur Ludovic Radeau, Monsieur Moi

Maillot d'homme, casquette et gilet,

Descendent les marches de l'escalier.

Monsieur et Monsieur se rendent à la salle du conseil (...). Importants.

Monsieur et Monsicur

Radeau Ludovic

Moi... moi! ?3 $^{33}$

La redondance des marques identitaires évoque un dédoublement. Cette impression est accrue par le patronyme du compagnon du narrateur. L'abréviation usuelle de son prénom Ludovic - Ludo appelle l'association avec l'adjectif «ludique»; Ludo - jeu- je? Il est intéressant de citer à cette occasion Pierre Mertens qui, dans une étude, écrit: «Le Moi Je est un Moi-jeu, mais où le ludisme peut être mélancolique et mortel ${ }^{34}$. Il est à remarquer d'ailleurs que le prénom de Ludovic est une version d'origine slave de Louis, phonétiquement proche du pronom personnel: lui, ce qui accentue encore davantage le thème du double. L'accent mis sur l'origine étrangère du personnage souligne cette altérité dont l'auteur se sent habité et qui n'est pas sans rappeler Leopoldus et Rodrigo, compagnons étrangers des narrateurs detreziens.

Le patronyme de l'Ami est éloquent, lui aussi. Radeau, engin de sauvetage, ce personnage l'est effectivement pour le narrateur en tant que l'antidote à l'influence nocive de la mère. A un autre niveau, ce personnage fonctionne comme la cristallisation des aspirations et des rêves du narrateur. Ludo incarne la joie de vivre, la liberté, il est le guide et l'initiateur, notamment dans la sexualité. Il est le seul à faire face à la mère, celle du narrateur, c'est lui qui sort victorieux du duel final pour périr à son tour ${ }^{35}$. Sur le plan symbolique c'est un affranchissement du «moi» du natrateur de l'emprise claustrale du monde extérieur, personnifié par la mère, et des obsessions intérieures personnifiées par Ludo. La fin du livre est explicite: le processus d'identification du narrateur-personnage principal s'accomplit, le «moi» du narrateur parvient à recouvrir son autonomisation: «La clé creuse un trou si profond que l'île s'y précipite, et les eaux, les maisons de la rue et même un morceau du ciel. Ludo me dépasse, file vers le fond. J'attrape au vol une bulle rouge, la dernière. Je la garde serrée dans ma main pour l'instant où je le retrouverais ${ }^{36}$.

Pour Conrad Detrez autobiographe, ce premier livre est de première importance. Il permet une lente émergence du Moi; d'abord flou et incertain, à la fin du livre il parvient à conquérir une autonomie que les livres suivants vont développer.

${ }^{33}$ Idem, p. 34.

${ }^{34}$ P. Mertens, Du retour à l'autobiographie, in: «Revue de L'Institut de Sociologie», ULB, Bruxelles 1990-1991, p. 62.

${ }^{35}$ Sur l'interprétation de ce personnage voir A.-J. Dubois, Lecture de Ludo, déjà cité p. 174.

${ }^{36}$ C. Detrez, Ludo, op. cit., p. 163. 
Ce processus de la lente prise de conscience du Moi du narrateur s'accompagne de l'appropriation de l'espace géographique. Comme le nom du narrateur n'est jamais mentionné, de même le texte ne contient aucune indication toponymique sauf le nom du fleuve: le Geer. Si l'identification est possible, c'est d'une part grâce au nom du fleuve et à la proximité de Maastricht, d'ailleurs ville d'origine des parents de Ludo; d'autre part c'est à cause des symboles tels que la platitude, l'atmosphère feutrée de gris, de froid et d'humidité qui évoquent le paysage nordique. C'est l'eau, appelé par un critique l'eau de la pluie nationale, qui est un élément dominant du paysage. L'eau, d'ailleurs complice de la mère, participe à la fermeture de l'espace; pour le protagoniste qui ne sait pas nager, l'eau devient un obstacle insurmontable. L'eau est également le symbole du danger: la pluie associée à la guerre, l'eau comparée au déluge au sens biblique du terme: «il y a l'eau. La rivière a noyé les chemins. Les chars s'enfoncent, les gens s'engloutissent, les plantes disparaissent de la surface du globe» ${ }^{37}$, elle est cet élément qui amène la mort comme châtiment, mais en même temps la délivrance (c'est dans les eaux que la mère et Ludo se perdent). Le fait que l'eau est étroitement associée à la mère, (elle est flamande) m'invite à risquer une hypothèse, contradictoire avec celle de Dubois qui y voit l'image de la Wallonie rurale, que ce climat grisâtre et froid n'est autre que le paysage flamand, terre matricielle, nourricière mais en même temps dévoratrice et destructrice. Y rester égale s'y noyer.

Les Plumes du coq est un second volet qui atteste de la progression dans l'élucidation du Moi du narrateur, déjà homodiégétique. Pour s'en persuader, il suffit de comparer la première phrase de Ludo («Ils disent tous que les eaux se sont arrêtées. (...) Ma mère annonce à tout le monde...») où le «je» n'apparaît que dans la seizième phrase, après l'évocation du monde extérieur, avec celle des Plumes du cog: «De ma vie je n'ai avancé sur un sol aussi gras».

Le Je du héros de ce livre, adolescent de 13 ans, s'affirme avec une force naissante qui, toutefois, sera exposée à de dures épreuves (manifestation, fuite du collège) et quelques défaillances symbolisées par l'épisode de l'entorse de la cheville. Certes, le personnage de l'Ami, qui s'appelle cette fois-ci Victor, est toujours présent. Il remplit le même rôle que Ludo auprès du narrateur: celui de séducteur. Toutefois il perd le rôle d'initiateur à la liberté; bien au contraire: Victor est le gardien des valeurs conservatrices: rester au collège, dans le pays. Il s'oppose donc au désir d'évasion du narrateur qui s'y adonne. Bien que l'impact de l'Ami sur le protagoniste soit encore remarquable, celui-ci parvient déjà à disposer de soimême sans arriver pour autant à décliner son identité. La saisie du Moi va de pair avec la modification de l'élément naturel; à la fluidité de l'eau succède la boue, caractéristique de la Wallonie. Le cadre géographique est précisé, le village est nommé: Saint-Trudon (vrai: Saint-Trond), de nombreux repères topographiques sont donnés: Bruxelles, Hasselt, Tournai; les Wallons et les Flamands sont évoqués,

\footnotetext{
${ }^{37}$ Idem, p. 74.
} 
même le mot «belge» apparaît dans la fameuse citation de César: horum omnium fortissimi sunt Belgae que les collégiens apprennent par cœur et qui est révélatrice de la nature du patriotisme enseigné. Ce qui achève l'identification du lieu et du temps de l'action, ce sont les événements historiques rapportés: la question royale, la manifestation à Grâce-Berleur ${ }^{38}$. La dimension irréelle de l'histoire traduit d'une part le refus de l'auteur de l'affronter en face, d'autre part, le refus du narrateur homodiégétique de s'y identifier. Car la boue, symbole de la Wallonie, est dangereuse: elle limite l'homme dans sa course à la liberté, elle l'engloutit, l'ensevelit (p. 54). Le symbole du coq n'est pas plus optimiste: enfermé, il se fait déplumer par les hommes du pouvoir, il se fait même violer par certains... prêtres. La Wallonie est ici associée au père, absent dans Ludo, multiplié dans Les Plumes du coq: "Il parait que mon père est parti comme je devais le faire: sans avertir. (...) Le prêtre lui (à la mère) conseilla de me conduire au collège. Là, j'aurais au moins vingt pères pour s'occuper de moi ${ }^{39}$; d'ailleurs le déplumage des poules renvoie également au métier du père ${ }^{40}$ de Detrez.

Quitter la Mère et le Père symboliques revient à accéder à l'âge adulte, à la maturation du Moi, atteinte dans le troisième livre, L'Herbe à brûler.

Dans le troisième volet de la trilogie le nom du narrateur est livrée pour la première fois établissant l'identité entre l'auteur, le narrateur et le personnage principal, sans que la latinisation de Conrad en «Conradus» au début du livre nuise à l'identification ${ }^{41}$ avec le nom sur la couverture. Elle est accrue par la reconnaissance explicite des origines du narrateur autodiégétique: «je suis Belge, fais-je, étonné de m'entendre décliner pour la première fois ma citoyenneté» ${ }^{42}$.

Cette auto-définition du début du livre est suivie de l'analyse basée sur l'observation, critique, de la réalité socio-politique belge sur l'exemple de Louvain et se termine par la négation et le rejet de la Belgique, manifestés par le départ pour le Brésil. La quête identitaire jusqu'alors parallèle à la quête nationale, subit la loi de proportionnalité inverse: le narrateur autodiégétique s'affirme plus fort à mesure qu'il s'éloigne, au sens psychologique et physique du terme, du pays.

Le titre semble d'ailleurs l'indiquer. Dubois interprète ce titre en se référant à la bible et au buisson ardent. Pourtant l'herbe, c'est aussi le fruit de l'eau et de la terre grasse, qui ne s'y acclimatant pas, est à brûler dans le feu du châtiment, de la purgation aussi donnant lieu à une renaissance.

Le début du livre qui relate, sur un mode poétique, la mort symbolique, le passage d'une étape de la vie à une autre, nouvelle, permet de considère le texte comme le bilan de la vie, d'une étape importante. De même, la première phrase du

${ }^{3 B}$ Idem, Les Plumes ..., op. cit., p. 137.

${ }^{39}$ Idem, L'Herbe ..., op. cit., p. 19-21.

${ }^{40}$ "Dans cet univers une chose me répugnait: le métier de mon père. Cet homme égorgeait des porcs, des moutons», idem, Le jardin de ma vie, dans la Lecture de Ludo, déjà cité, p. 196.

${ }^{41}$ "Les humanistes latinisent toujours leur nom», idem, L'Herbe ..., op. cit., p. 28.

${ }^{42}$ Idem, p. 30. 
récit suggère la dimension introspective: «est-ce à cause du parfum des plantes? Mon âme s'est souvenue de ce jour où elle a quitté mon corps pour la première fois. C'était un dimanche.... ${ }^{43}$. La structure du livre: reprise des étapes décrites dans les livres précédents, le regard rétrospectif, la fin qui s'articule autour du renouveau symbolisé par le retour à la maison maternelle, consacre le terme de trilogie autobiographique et participe dans ce projet globalisant de l'existence du narrateur.

Le jeu avec le «je» serait également une autre façon d'exprimer cette attitude ambiguë qu'adopte l'auteur envers son pays natal. La Belgique, on l'a vu, n'inspire que son ironie: "Au royaume de sire Baudouin (et Mme Fabiole) l'insurrection n'est pas inscrite à l'ordre du jour. On prévoit plutôt qu'il pleuvra» ${ }^{44}$. La distance, tant psychique que géographique, l'éloignement, l'exil enfin; autant de solutions que trouve Detrez, cet «apatride mental» ${ }^{45}$ pour régler ses rapports avec le pays. Pour lui, l'essentiel est: «de se retrouver de l'autre coté, dans l'autre pays» ${ }^{46}$ pour mieux pouvoir observer, voir: «Regarder au-dessus de soi, en soi: on trouve la même chose» ${ }^{47}$.

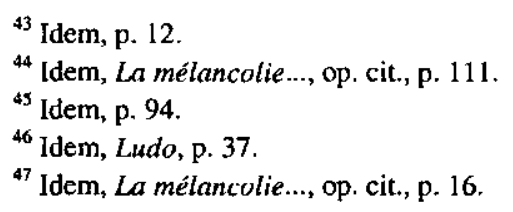

